\title{
Molecular heterogeneity in hepatocellular carcinoma
}

\author{
Shijia Zhu ${ }^{1}$ \& Yujin Hoshida*,1 \\ ${ }^{1}$ Liver Tumor Translational Research Program, Harold C. Simmons Comprehensive Cancer Center, Division of Digestive and Liver \\ Diseases, Department of Internal Medicine, University of Texas Southwestern Medical Center, Dallas, TX 75390, USA \\ *Author for correspondence: Yujin.Hoshida@UTSouthwestern.edu \\ ${ }^{6}$ clinical trials suggest that subtype-targeted therapy may be a viable option of medical therapy in \\ advanced HCC"
}

First draft submitted: 8 July 2018; Accepted for publication: 17 July 2018; Published online: 11 September 2018

Keywords: clinical trial • hepatocellular carcinoma • molecular classification • molecular targeted therapy

Recent successful clinical trials have led to or likely lead to the regulatory approval of several molecular targeted agents for first- and second-line treatment of hepatocellular carcinoma (HCC), including multikinase inhibitors, lenvatinib, regorafenib and cabozantinib, as well as immune checkpoint inhibitors nivolumab and pembrolizumab [1-5]. However, objective response rates to these agents are only $20 \%$ at maximum and patient survival benefit is no more than a few months despite the high cost of the drugs. A recent simulation-based analysis has reported that regorafenib treatment for advanced-stage HCC patients is not cost-effective with an incremental cost-effectiveness ratio (ICER) of $\$ 224,396$ per quality-adjusted life year gained, which far exceeds the widely accepted incremental cost-effectiveness ratio cutoff of $\$ 50,000$ [6]. Given that only small proportion of the patients respond to each therapy, it is critical to identify potential responders to avoid prescribing the drugs to patients who will not benefit from the treatment and enable cost-effective patient management [7]. However, no biomarker of drug response is available for any of the approved drugs for HCC to date.

\section{Elusive therapeutic targets in HCC}

Somatically mutated oncogenic kinases have been the major target of pharmacological intervention in solid cancers. However, such therapeutically more accessible, so-called 'druggable', genes or proteins (e.g., EGFR) are rarely mutated in HCC, whereas therapeutically unamenable genes such as TP53, CTNNB1, ARID1/2, TERT and $A L B$ are most frequently mutated in HCC [8]. Oncogenic genomic DNA amplification in FGF19 has also been explored as a point of intervention by molecular targeted agents, although frequency of the event is less than $5 \%$ [9]. The rarity of such somatic DNA structural aberrations makes the planning and conduct of clinical trials more challenging due to the requirement of screening many patients to enroll a sufficient number of potential responders with the molecular aberration of interest.

Functional molecular pathway dysregulation is often shared with a larger fraction of HCC tumors compared with the somatic DNA aberrations. For example, the c-Met pathway is aberrantly activated in approximately one-fourth of HCC tumors [7]. A small molecule compound, tivantinib, has been tested as a c-Met inhibitor for second-line treatment of advanced HCC in a Phase II trial, in which positive c-Met immunostaining was associated with extended patient survival. In a subsequent Phase III trial, c-Met positivity was not validated as a biomarker of tivantinib response [10] and more recent studies suggest that the compound actually kills HCC cells positive for cell proliferation markers such as Ki-67, which could be correlated with c-Met expression [11].

Another example is ramucirumab, a monoclonal antibody against VEGF receptor type, which was tested as a second-line therapy for advanced HCC in a Phase II trial (REACH trial) [12]. Despite the absence of overall survival benefit in all patients enrolled and evaluated in the trial, patients with serum $\alpha$-fetoprotein (AFP) level greater than $400 \mathrm{ng} / \mathrm{ml}$ showed statistically significant improvement of overall survival in a post hoc subgroup analysis. This 
finding was subsequently confirmed in a Phase III trial (REACH-2 trial, NCT02435433), although it is unclear why AFP-positive HCC tumors respond to the drug.

While the studies underscore difficulty in verifying the molecular mechanism of drug action in an actual clinical setting, these empirical observations support the notion that a subset of HCC cells or tumors harboring certain functional pathway dysregulations, preferentially respond to a specific drug.

\section{Molecular intertumor heterogeneity as potential guide for individualized HCC therapy}

Genome-wide molecular profiling of clinical HCC tissues has identified heterogeneous molecular aberrations across the tumors, many of which are shared by subgroup of tumors and form molecular subtypes of HCC tumors [7]. A meta-analysis of transcriptome profiles from multiregional, multiracial and ethnic patient cohorts identified aggressive and indolent molecular subtypes of HCC tumors [13]. A subset of the aggressive tumors are characterized by overexpression of stemness marker genes and proteins such as EPCAM, activation of specific oncogenic pathways such as Myc and Akt pathways, and accompanied with high serum AFP levels (S2 subtype) compared with other tumors. AFP-negative aggressive tumors are more frequently accompanied with immune cell infiltrates (S1 subtype) [14,15]. Presence of the subtypes has been confirmed in independent studies utilizing multiomics datasets such as coding/noncoding transcriptome, genetic mutation and epigenome profiles [8,16]. From the molecular subtype perspective, ramucirumab may elicit anti-HCC effect through some of the molecular pathways specifically dysregulated in the AFP-expressing subtype.

It could be challenging to unequivocally elucidate how the mechanism of a molecular targeted agent kills HCC cells, given the highly complex genomic aberrations and tumor microenvironment in clinical HCC. However, identifying such subtype-specific drug response may have a significant clinical utility by enabling clinical trials enriched for certain subtype, which is more prevalent than somatic DNA mutations in druggable genes [7]. Such subtype-enriched clinical trial will indeed substantially reduce the number of subjects to be enrolled (up to fourfold reduction), while achieving sufficient statistical power to detect expected therapeutic benefit in clinical trials [14]. In fact, such subtype-based management has been successfully utilized in other cancer types such as HER2-positive gastric cancer and estrogen receptor-positive breast cancer.

\section{Molecular subtype-guided preclinical drug discovery}

Studies have shown that global transcriptome profiles classify human HCC cell lines into two groups according to AFP expression levels [17]. Furthermore, the two subgroups mimic transcriptional dysregulation observed in either or the AFP-positive (S2) or negative (S1) HCC subtypes [17]. Of note, these subgroups of HCC cell lines show a distinct response to molecular targeted agents. For example, a small molecule Src/Abl kinase inhibitor, dasatinib, showed more preferential killing of the S1-like cell lines, whereas MYC pathway-activated S2-like cell lines showed higher sensitivity to a small molecule BET bromodomain inhibitor, (+)-JQ1, which has anti-MYC activity [17,18]. These studies suggest that the panel of HCC cell lines can be used to screen compounds with subtype-specific efficacy like ramucirumab. Other in vitro systems, such as patient-derived cell lines and patient-derived xenografts, may also be used for similar application with determination of the molecular subtype of each tumor line at baseline as well as for potential change over time after the drug treatment $[19,20]$. Molecular subtype-specific drug effect can be indeed monitored in in vivo HCC models [21].

\section{Intratumor heterogeneity may complicate therapeutic decision making}

Clinically, it is well known that an HCC tumor nodule often presents a nodule-in-nodule appearance with histologically distinct subcomponents [14]. Interestingly, histological variants represented by each subcomponent (e.g., a micro- or macro-trabecular pattern, clear cell appearance, pseudoglandular pattern and steatohepatitic appearance) are correlated with presence of the molecular HCC subtypes [14]. This finding was confirmed in independent studies from Europe and Asia [15,22]. Furthermore, finer resolution analyses, down to single celllevel, for example, have revealed intratumor heterogeneity in genetic aberrations, transcriptional and epigenetic dysregulation, protein abundance and stemness marker gene expression, which can be utilized to infer the path of clonal evolution and origin for each tumor cell population [23]. Characterization of infiltrating immune cells provides another layer of information to determine comprehensive landscape of intratumor heterogeneity [15].

In the clinical setting, such intratumor heterogeneity, specifically presence of multiple tumor cell populations with distinct molecular targets, likely obscures therapeutic strategy with the use of selective molecular targeted agents. Moreover, accurate and sensitive detection of the molecular aberrations will be critical to avoid therapeutic 
decision making based on false negative detection of the targeted molecular features. The major logistical challenge has been the lack of access to HCC tissue specimens in daily clinical care of the patients with advanced-stage HCC subjected to medical therapies. Body fluid-based assessment, so-called liquid biopsy, of tumor-derived biomolecules such as circulating tumor cells (CTC), cell-free DNA, and exosomal noncoding RNA may provide access to the information without relying on acquisition of tissue [24]. However, recent studies have noted several major limitations that should be addressed to clarify their real clinical utility and specific scenarios of application. For example, detected circulating tumor cells populations can significantly vary according to the sites of blood sampling [25]. Sensitivity of circulating cell-free DNA-based detection of somatic DNA mutations found in tissue is less than 50\% even with deep sequencing of targeted genes [24]. In addition, these biomolecules may be released into bloodstream from specific subpopulation of tumor cells that are prone to die, reside closely to vascular systems, or have other properties, and therefore may not serve as representative readout of a whole tumor nodule. Even in tissue-based assessment, only limited portion of a tumor nodule is analyzed. Imaging-based assessment may overcome the hurdle by noninvasively capturing the information of intratumor heterogeneity throughout an entire nodule. Quantitative analysis of mutiparametric MRI can be used to evaluate intratumor heterogeneity in an HCC nodule, which is correlated with expression of therapeutically relevant genes such as FGFR4 and PDCD1 [26]. Pretreatment contrast-enhanced CT texture parameters was associated with prognosis after sorafenib treatment [27].

\section{Conclusion \& future perspective}

'One-size-fits-all' medical therapy yields limited patient survival benefit despite the high drug costs. The ever expanding capability to molecularly characterize inter- and intratumor heterogeneity is expected to facilitate development of rational and individualized treatment strategies in HCC. However, detection sensitivity and specificity of body fluid- or imaging-based modalities should be improved to support clinical decision making and enable clinical trials enriched for possible responders to targeted agents. Recent observations in Phase III clinical trials suggest that subtype-targeted therapy may be a viable option of medical therapy in advanced HCC, like other cancer types. Experimental models of the HCC subtype may assist development of subtype-targeted medical therapies in HCC.

Financial \& competing interests disclosure

Y Hoshida is supported by NIH/NIDDK DK099558, European Union ERC-2014-AdG-671231 HEPCIR, Irma T Hirschl Trust, US Department of Defense W81XWH-16-1-0363.

The authors have no other relevant affiliations or financial involvement with any organization or entity with a financial interest in or financial conflict with the subject matter or materials discussed in the manuscript apart from those disclosed.

No writing assistance was utilized in the production of this manuscript.

\section{Open access}

This work is licensed under the Attribution-NonCommercial-NoDerivatives 4.0 Unported License. To view a copy of this license, visit http://creativecommons.org/licenses/by-nc-nd/4.0/

\section{References}

1. Kudo M, Finn RS, Qin S et al. Lenvatinib versus sorafenib in first-line treatment of patients with unresectable hepatocellular carcinoma: A randomised phase 3 non-inferiority trial. Lancet 391(10126), 1163-1173 (2018).

2. Bruix J, Qin S, Merle P et al. Regorafenib for patients with hepatocellular carcinoma who progressed on sorafenib treatment (resorce): A randomised, double-blind, placebo-controlled, phase 3 trial. Lancet 389(10064), 56-66 (2017).

3. Abou-Alfa GK, Meyer T, Cheng AL et al. Cabozantinib in patients with advanced and progressing hepatocellular carcinoma. N. Engl. J. Med. 379(1), 54-63 (2018).

4. El-Khoueiry AB, Sangro B, Yau T et al. Nivolumab in patients with advanced hepatocellular carcinoma (checkmate 040): An open-label, non-comparative, phase 1/2 dose escalation and expansion trial. Lancet 389(10088), 2492-2502 (2017).

5. Zhu AX, Finn RS, Edeline J et al. Pembrolizumab in patients with advanced hepatocellular carcinoma previously treated with sorafenib (keynote-224): a non-randomised, open-label phase 2 trial. Lancet Oncol. 19(7), 940-952 (2018).

6. Parikh ND, Singal AG, Hutton DW. Cost effectiveness of regorafenib as second-line therapy for patients with advanced hepatocellular carcinoma. Cancer 123(19), 3725-3731 (2017).

7. Goossens N, Sun X, Hoshida Y. Molecular classification of hepatocellular carcinoma: potential therapeutic implications. Hepat. Oncol. 2(4), 371-379 (2015). 
8. Cancer Genome Atlas Research Network. Electronic address wbe, cancer genome atlas research n. comprehensive and integrative genomic characterization of hepatocellular carcinoma. Cell 169(7), 1327-1341 e1323 (2017).

9. Hagel M, Miduturu C, Sheets $\mathrm{M}$ et al. First selective small molecule inhibitor of fgfr 4 for the treatment of hepatocellular carcinomas with an activated fgfr4 signaling pathway. Cancer discov. 5(4), 424-437 (2015).

10. Rimassa L, Assenat E, Peck-Radosavljevic M et al. Tivantinib for second-line treatment of met-high, advanced hepatocellular carcinoma (metiv-hcc): a final analysis of a phase 3, randomised, placebo-controlled study. Lancet Oncol. 19(5), 682-693 (2018).

11. Rebouissou S, La Bella T, Rekik S et al. Proliferation markers are associated with met expression in hepatocellular carcinoma and predict tivantinib sensitivity in vitro. Clin. Cancer Res. 23(15), 4364-4375 (2017).

12. Zhu AX, Baron AD, Malfertheiner $P$ et al. Ramucirumab as second-line treatment in patients with advanced hepatocellular carcinoma: analysis of reach trial results by child-pugh score. JAMA Oncol. 3(2), 235-243 (2016).

13. Hoshida $\mathrm{Y}$, Nijman SM, Kobayashi $\mathrm{M}$ et al. Integrative transcriptome analysis reveals common molecular subclasses of human hepatocellular carcinoma. Cancer Res. 69(18), 7385-7392 (2009).

14. Tan PS, Nakagawa S, Goossens $\mathrm{N}$ et al. Clinicopathological indices to predict hepatocellular carcinoma molecular classification. Liver Int. 36(1), 108-118 (2016).

15. Kurebayashi Y, Ojima H, Tsujikawa $\mathrm{H}$ et al. Landscape of immune microenvironment in hepatocellular carcinoma and its additional impact on histological and molecular classification. Hepatology doi: 10.1002/hep.29904. (2018). (Epub ahead of print)

16. Chaisaingmongkol J, Budhu A, Dang $\mathrm{H}$ et al. Common molecular subtypes among asian hepatocellular carcinoma and cholangiocarcinoma. Cancer Cell 32(1), 57-70 e53 (2017)

17. Hirschfield $\mathrm{H}$, Bian CB, Higashi $\mathrm{T}$ et al. In vitro modeling of hepatocellular carcinoma molecular subtypes for anti-cancer drug assessment. Exp. Mol. Med. 50(1), e419 (2018).

18. Finn RS, Aleshin A, Dering J et al. Molecular subtype and response to dasatinib, an src/abl small molecule kinase inhibitor, in hepatocellular carcinoma cell lines in vitro. Hepatology 57(5), 1838-1846 (2013).

19. Gao Q, Wang ZC, Duan M et al. Cell culture system for analysis of genetic heterogeneity within hepatocellular carcinomas and response to pharmacologic agents. Gastroenterology 152(1), 232-242 e234 (2017)

20. Fong ELS, Toh TB, Lin QXX et al. Generation of matched patient-derived xenograft in vitro-in vivo models using $3 \mathrm{~d}$ macroporous hydrogels for the study of liver cancer. Biomaterials 159, 229-240 (2018).

21. Schmidt B, Wei L, Deperalta DK et al. Molecular subclasses of hepatocellular carcinoma predict sensitivity to fibroblast growth factor receptor inhibition. Int. J. Cancer 138(6), 1494-1505 (2016).

22. Calderaro J, Couchy G, Imbeaud $S$ et al. Histological subtypes of hepatocellular carcinoma are related to gene mutations and molecular tumour classification. J. Hepatol. 67(4), 727-738 (2017).

23. Zhai W, Lim TK, Zhang $\mathrm{T}$ et al. The spatial organization of intra-tumour heterogeneity and evolutionary trajectories of metastases in hepatocellular carcinoma. Nat. Commun. 8, 4565 (2017).

24. Huang A, Zhao X, Yang XR et al. Circumventing intratumoral heterogeneity to identify potential therapeutic targets in hepatocellular carcinoma. J. Hepatol. 67(2), 293-301 (2017).

25. Sun YF, Guo W, Xu Y et al. Circulating tumor cells from different vascular sites exhibit spatial heterogeneity in epithelial and mesenchymal composition and distinct clinical significance in hepatocellular carcinoma. Clin. Cancer Res. 24(3), 547-559 (2018).

26. Hectors $\mathrm{SJ}$, Wagner $\mathrm{M}$, Bane $\mathrm{O}$ et al. Quantification of hepatocellular carcinoma heterogeneity with multiparametric magnetic resonance imaging. Sci. Rep. 7(1), 2452 (2017).

27. Mule S, Thiefin G, Costentin C et al. Advanced hepatocellular carcinoma: Pretreatment contrast-enhanced CT texture parameters as predictive biomarkers of survival in patients treated with sorafenib. Radiology 288(2), 445-455 (2018). 